\title{
A systematic approach to report, categorize and grade quality failures in clinical biochemistry laboratory - 'single center analysis'
}

\author{
Bhavesh R. Sadariya ${ }^{1}$, Boinapalli Sudhakar ${ }^{2, *}$ \\ ${ }^{1}$ Assistant Professor, ${ }^{2}$ Associate Professor, Dept. of Biochemistry, Pacific institute of medical sciences, Umarada, \\ Udaipur, Rajasthan, India
}

*Corresponding Author: Boinapalli Sudhakar

Email: boinapallisudhakar@gmail.com

Received: $27^{\text {th }}$ March, 2018

Accepted: $15^{\text {th }}$ June, 2018

\begin{abstract}
Introduction: Quality failures in clinical biochemistry laboratory have an adverse impact on patient care. Identification and correction of the quality failures are essential to decrease risk and improve patient safety in hospital.

Materials and Methods: This study was assessed over 14 months from January 2017 to February 2018. A designed 'Quality enquiry forms' were introduced to report quality failures which prioritize corrective action. Quality failures were categorized in three phases: preanalytical, analytical and postanalytical with subcategories in each phase. Quality failures were graded on the basis of actual ('A' score) and potential ('P' score) adverse outcome on patient care using 5 point scoring system.

Results: The numbers of quality failures reported were $453(0.53 \%)$ out of 84,569 requisites received by the clinical biochemistry laboratory, during this entire study period. Quality failures were reported as 50.3\%, 5.1\% and $44.6 \%$ in preanalytical, analytical and postanalytical phases respectively. Quality failure severity 'A' score was tilted towards lower side: $58.5 \%$ allocated an 'A' score of 1 (least severe grade), while for the 'P' score towards higher side: $45.5 \%$ allocated a 'P' score of 5 (most severe grade).

Conclusion: Quality enquiry reporting system is an effective method for recognition of quality failures in clinical biochemistry laboratory. Systematic approach in this study encompasses a multifaceted strategy for error analysis based on error detection and prevention.
\end{abstract}

Keywords: Quality failure, Systematic approach, Error, Clinical biochemistry laboratory.

\section{Introduction}

Clinical biochemistry laboratory plays a key role in the diagnosis of patients. About $80-90 \%$ of diagnoses are made on the basis of laboratory test results. Frequency of laboratory errors reported in range 0.012$0.6 \%{ }^{1}$ Clinical biochemistry laboratory testing process is a complex process, which begins with the test selection and ends with the return of a timely, accurate and correctly interpreted result to the clinician. ${ }^{2}$ Laboratory testing involves different procedures, equipment, technology and human skills. Error can occur at any phases of laboratory testing process. Effective patient treatment and safety can be improved by detecting and preventing the laboratory errors at the time of their occurrence. ${ }^{3}$ The International Organization for Standardization suggest to implement quality management system in clinical biochemistry laboratories for improving patient safety. ${ }^{4}$ Identification of laboratory failures helps to find out high risk prone areas and to prioritize corrective actions.

Previously, different terms were used for laboratory errors, like blunders, errors, mistakes, problems, without any established definition. ${ }^{5-8}$ "Quality failure" is the appropriate to reduce the sense of blame among staff and support them to report the errors. ${ }^{1}$ Quality failure is defined as any failure to attain the desired output quality necessary for effective patient care during any phases of laboratory process. ${ }^{2}$
Occurrence of laboratory errors can be scrutinized by mainly two paradigms: i) Person approach ii) System approach. Errors originate mainly from human resource implies person approach while system approach indicates errors concerned with faulty system. ${ }^{3,9}$ If there is no systematic method for the identification of quality failures, errors will go unrecognized with missing corrective actions. Many clinical biochemistry laboratories do not have established system to monitor quality failures for routine practice.

This study was designed to build up a systematic approach to recognize, classify, grade and prevent the quality failures in clinical biochemistry laboratory for routine practice.

\section{Materials and Methods}

This study was conducted at clinical biochemistry laboratory, Pacific institute of medical sciences, Umarda, Udaipur over a period of 14 months from January 2017 to February 2018. The study was approved by institutional ethics committee of PIMS, Udaipur. Clinical biochemistry laboratory receives around 6040 requests (samples) per month. Laboratory process includes receiving of labelled samples with requisition form, centrifugation and analysis of samples in fully automated analyzers, manually transforming the results from analyzer to laboratory information system and approval of reports. 
Quality enquiry form was prepared and circulated among all work areas in the clinical biochemistry laboratory. Quality failures identified in any step of laboratory procedure was reported in quality enquiry form under the following headings: Description of failure recognized, cause for quality fail, appropriate action to be taken. All the quality enquiry forms were scrutinized by senior member of clinical biochemistry laboratory. Wherever needed quality failure reports were discussed with clinical staff to assess the adverse outcome on patient care. Quality failures were classified based upon the cause into three categories: Pre analytical, analytical and post analytical. Quality failures were graded on the basis of actual and potential adverse outcome on patient care.
Categorization of Quality Failures: Three major categories were subdivided in context to the particular reason of failure (Table 1).

Scoring of Quality Failures: On the basis of adverse outcome on patient health care, quality failures were graded into actual ('A' score) and potential ('P' score). A score measures actual adverse clinical outcome of patient and $\mathrm{P}$ score measures worst potential adverse outcome of patient. 'A' score and 'P' score ranked using similar severity ranking scale by senior member of laboratory staff as shown in Table $2 .^{2}$

Fifty two quality enquiry reports were analyzed and scored by two laboratory staff members independently to validate inter-observer consistency for score distribution.

Table 1: Routinely reported quality failures

\begin{tabular}{|l|c|c|}
\hline \multicolumn{1}{|c|}{ Preanalytical phase } & \multicolumn{1}{c|}{ Analytical phase } & Post analytical phase \\
\hline $\begin{array}{l}\text { 1.Wrong sample type } \\
\text { a. Wrong tubes } \\
\text { b. Hemolysis/Lipemia } \\
\text { c. others (Sample withdrawn from } \\
\text { intravenous line) }\end{array}$ & $\begin{array}{c}\text { 1. Wrong analytical result } \\
\text { generated although satisfactory } \\
\text { quality control (QC) } \\
\text { 2.Wrong labelled sample or request } \\
\text { form }\end{array}$ & $\begin{array}{c}\text { 1. Failure to inform critical report } \\
\text { to clinian }\end{array}$ \\
\hline $\begin{array}{l}\text { 3.Wrong/missing sample ID } \\
\text { correction of internal quality } \\
\text { control (IQC) failure }\end{array}$ & 2. Transcription error \\
\hline 4.Sample spillage in laboratory & - & $\begin{array}{c}\text { 3. Non arrival of hard copy of } \\
\text { report to ward }\end{array}$ \\
\hline $\begin{array}{l}\text { 5.Sample misplaced in laboratory } \\
\text { 6.Wrong entry of patient or test } \\
\text { data on laboratory } \\
\text { information system (LIS) }\end{array}$ & - & 4. Calculation errors \\
\hline $\begin{array}{l}\text { 7. Incorrect centrifugation } \\
\text { improper storage of samples }\end{array}$ & - & $\begin{array}{c}\text { 5.Missing entry of investigation } \\
\text { value }\end{array}$ \\
\hline
\end{tabular}

Table 2: Grading of quality failures ${ }^{2}$

\begin{tabular}{|l|l|}
\hline Score & \multicolumn{1}{c|}{ Effect of quality failure } \\
\hline 1 & No adverse clinical outcome: Unchanged patient management \\
\hline 2 & $\begin{array}{l}\text { No adverse clinical outcome: Minor change in patient management } \\
\text { e.g. short delay in diagnosis due to delay in reporting test result }\end{array}$ \\
\hline 3 & $\begin{array}{l}\text { Minor adverse clinical outcome } \\
\text { e.g. need for an additional venepuncture }\end{array}$ \\
\hline 4 & $\begin{array}{l}\text { Moderate adverse clinical outcome } \\
\text { e.g. on basis of incorrect blood glucose result patient started on hypoglycemic medication }\end{array}$ \\
\hline 5 & $\begin{array}{l}\text { Significant adverse clinical outcome } \\
\text { e.g. significant morbidity, mortality }\end{array}$ \\
\hline
\end{tabular}

\section{Results}

Total 453 quality enquiry reports concluded against background of 84569 requests received by the laboratory, i.e. $0.53 \%$ of total requests, during this study. An average quality enquiry forms notified 32.4 per month. Total $398(87.6 \%)$ of all quality failures were reported by laboratory staff (through staff vigilance and existing quality verification) and remainders by service users. Forty one percentage of quality failures were notified by the laboratory staff prior to the release of the test results to the test prescribing clinicians. $50.3 \%$ and $44.6 \%$ of quality failures occurred in pre and post analytical phases respectively as depicted in Fig. 1. Fig. 2 shows two common causes of errors found were wrong sample types (31.3\%) and transcription errors (35.9\%). 
The quality enquiry reports analyzed by two laboratory staff members shows concordance in $96.1 \%$ and $80.1 \%$ quality failures for ' $\mathrm{A}$ ' score and ' $\mathrm{P}$ ' score respectively. Agreement between two observers were analysed by kappa statistics. ${ }^{10}$ A perfect agreement $(\mathrm{k}=0.866)$ for ' $\mathrm{A}$ ' score and substantial agreement $(\mathrm{k}=0.734)$ for ' $\mathrm{P}$ ' score were found between two observer scores.

The clustering pattern as depicted in Fig. 3 and Fig. 4 reflects lower ' $\mathrm{P}$ ' score in transcriptional errors and higher ' $\mathrm{P}$ ' score in wrong sample types respectively.

Severity grade for the 'A' score were tilted in low score direction, while for the ' $\mathrm{P}$ ' score in high score direction as depicted in Table 3. The percent quality failures resulted in unchanged patient management; no adverse clinical outcome and minor adverse clinical outcome were $58.5 \%$ ('A' score grade 1) and $29.4 \%$ ('A' score grade 3) respectively. $45.5 \%$ quality failures shows significant adverse clinical outcome having scored as 'P' grade 5.
Table 3: Percentage of severity grade for ' $A$ ' and ' $P$ ' scores of total quality failure

\begin{tabular}{|c|c|c|}
\hline Severity grade & A score & P score \\
\hline 1 & $58.5 \%$ & $31.8 \%$ \\
\hline 2 & $12.1 \%$ & $12.1 \%$ \\
\hline 3 & $29.4 \%$ & $6.0 \%$ \\
\hline 4 & $0 \%$ & $4.7 \%$ \\
\hline 5 & $0 \%$ & $45.5 \%$ \\
\hline
\end{tabular}

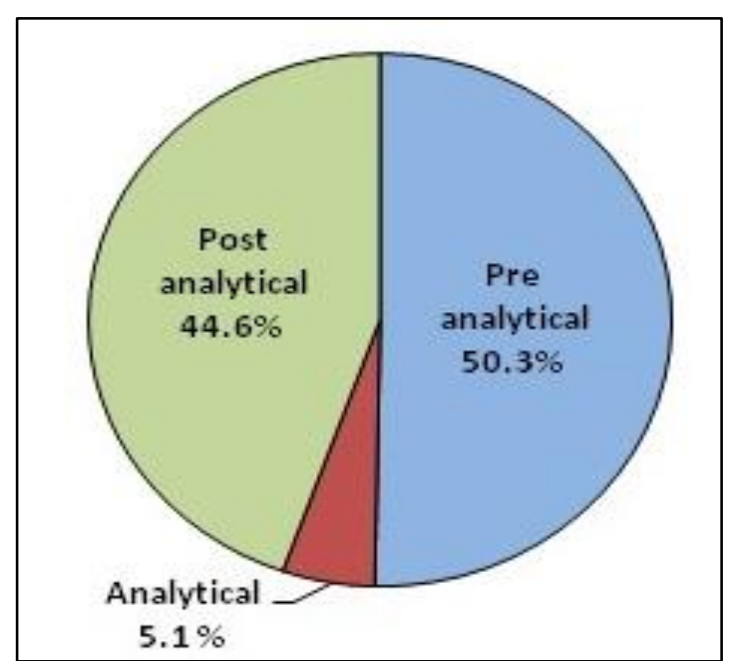

Fig. 1: Percentage of quality failures in three main phases

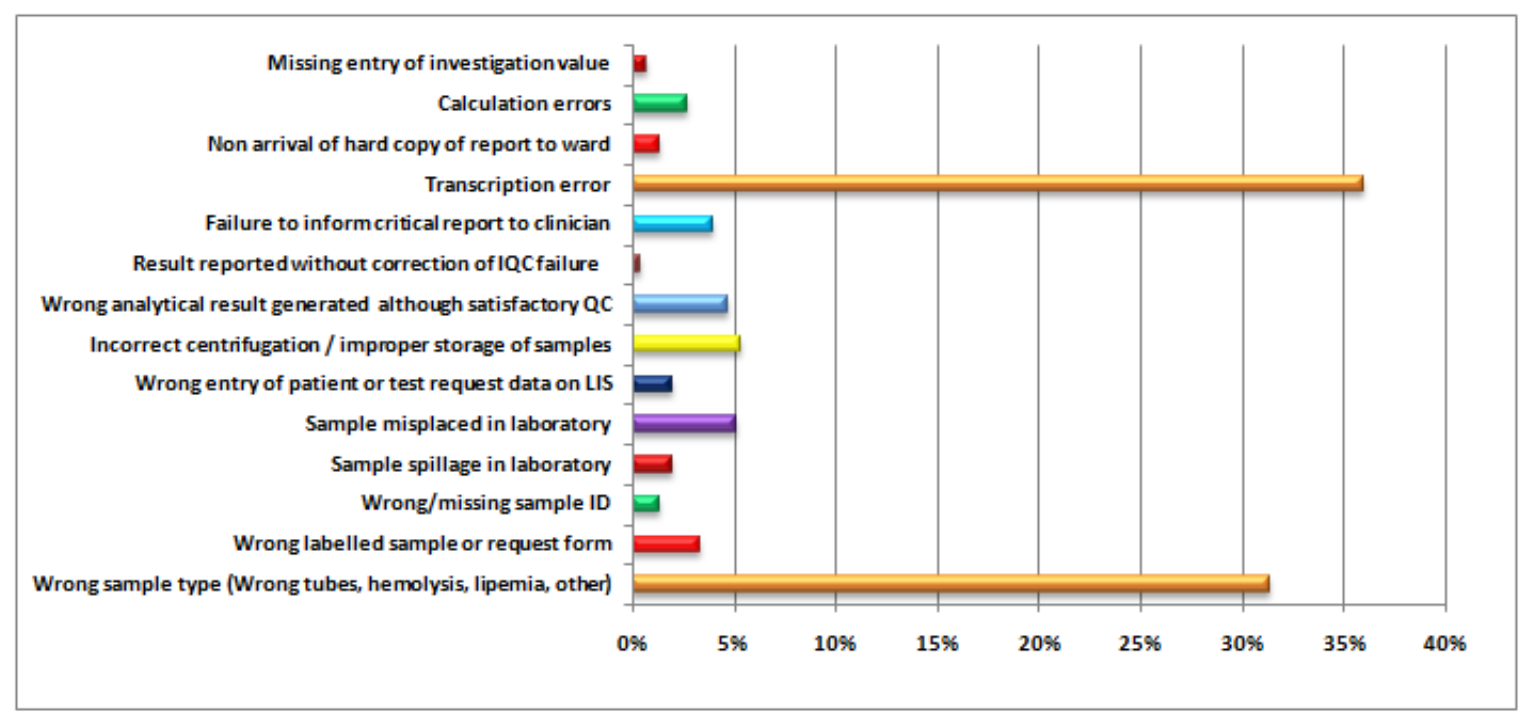

Fig. 2: Percentage of quality failures by cause 


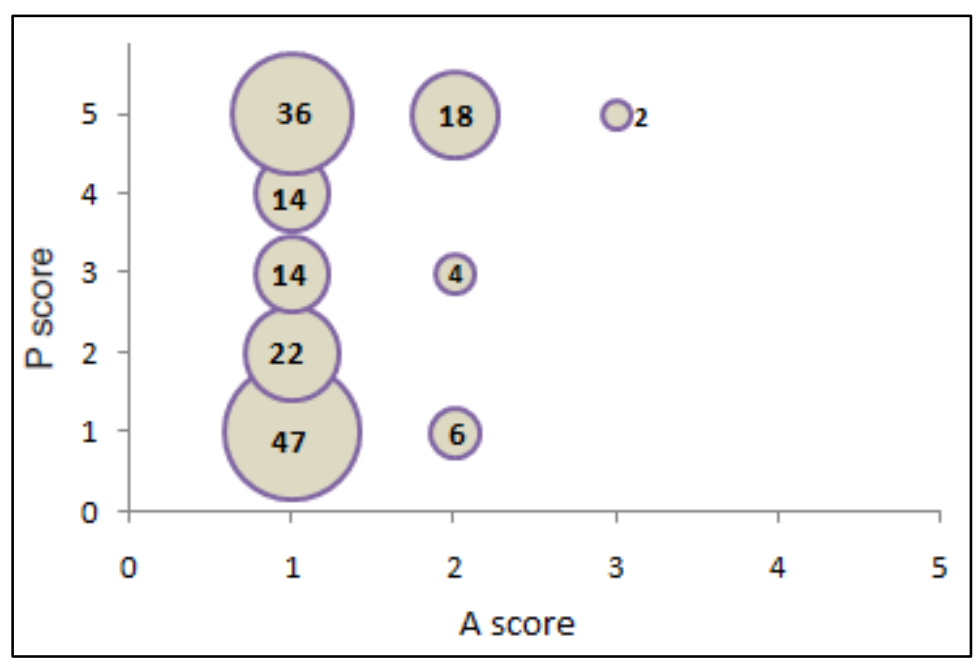

Fig. 3: Plots of A score against $P$ score for transcriptional errors

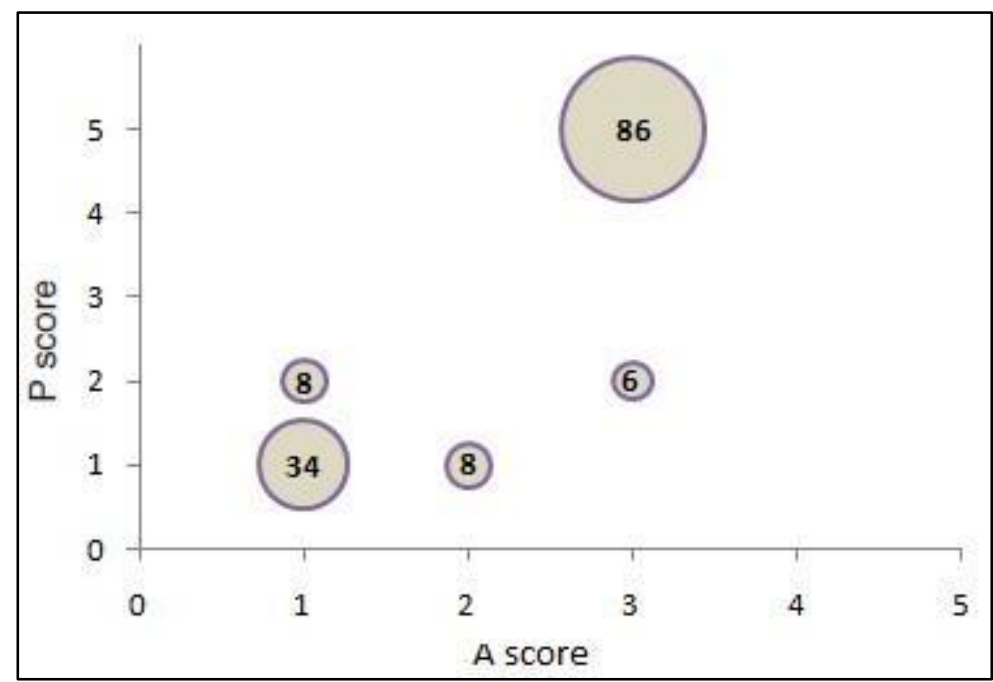

Fig. 4: Plots of A score against $P$ score for wrong sample types

\section{Discussion}

In this study a systematic approach used to recognize, classify and grade the quality failures in the clinical biochemistry laboratory. This approach reveals the term quality failure instead of laboratory errors/blunders/mistakes/problems is helpful to encourage the staff to report the errors. To augment the patient safety the identification of quality failure requires improved and constructive attitude to work by laboratory staff. The improved quality reporting system confirms easy to execute the details of actual or potential quality failures which provide equal opportunity to all the laboratory staff. Though the occurrence of reported quality failures was $0.53 \%$ of total requests received by the laboratory, it is within reported frequency of $0.12-0.6 \% .^{1}$ Identification of quality failures can be done by laboratory staff vigilance, spotting errors by laboratory service users and established policies like quality control monitoring, delta checks, appropriate maintenance of instruments, etc. Various staff is involved in the total testing process i.e. nurses involved in sample withdrawn, laboratory workers involved in sample process and analysis, ward boy involved in sample transportation. Therefore, frequent training of nurses and other laboratory workers is needed to be more specialized in taking blood samples and other interventions. ${ }^{11}$ Recurrence of the quality failures is reduced, if identification of basic causes and proper corrective actions taken.

The cause of quality failures classified into three broad categories namely preanalytical, analytical and postanalytical phases. Our study shows maximum quality failures arise in preanalytical $(50.3 \%)$ and postanalytical (44.6\%) phase, while minimal in analytical $(5.1 \%)$ phase. Studies conducted previously reported frequency of the quality failures occurs in the preanalytical phase (range 31.6-75\%), analytical phase (range 9-30.8\%) and postanalytical phase (range 13.3 $31.6 \%) .^{5-8,12}$ In present study postanalytical errors (44.6\%) found higher compared with previous studies, while analytical errors $(5.1 \%)$ were lesser. Possible reasons for reduced analytical errors may be due to 
effective quality control monitoring and automation in clinical biochemistry laboratory. Increased postanalytical errors (transcription errors) may be due to computer operator having less knowledge on investigations and values resulting in frequently interchanging values.

A simple 5 point grading was used to score actual (A) and potential (P) adverse effect of quality failures. Most common causes of quality failures have high potential severity as compared to actual adverse impact on patient (Fig. 3 and Fig. 4). The recognition of quality failures with high ' $\mathrm{P}$ ' score/low 'A' score contributes to review a relevant laboratory system as well as emphasize the importance of constant vigilance. The detection of quality failures with numerical values of actual and potential scores makes easy to recognize recurring errors and take earlier corrective action. In our study, recurrence of transcriptional errors can be reduced by sensitization of computer operator and laboratory staff regarding investigation's reference, critical and linearity values. Our study supports with a review study conducted in India highlights importance of identifying quality failures in various phases of clinical biochemistry laboratory testing process for requesting physician and to develop quality indicators for prevention..$^{13}$ our study also support with previous research which shows that pre analytical and post analytical constitute majority of the errors reason is due to lack of approach towards Phlebotomy. ${ }^{14}$

In this study, reporting of the quality failures was not completely performed during night shifts and holidays. More studies should be carried to find the frequency of quality failures occurred at different shifts i.e. morning, evening, night and at multi centres.

\section{Conclusion}

This study concludes quality enquiry reporting system is an effective systematic approach for recognization of quality failures and its actual/potential effect on patient care. Quality enquiry reports helps in identification of prompt risk areas for failure and focus to take preferred corrective action. So, practice of systematic approach is a fruitful way to recognize, classify, grade and prevent the quality failures in clinical biochemistry laboratory for routine work.

\section{Acknowledgement}

The authors are thankful to the technical staff of clinical biochemistry laboratory, Pacific Institute of Medical Sciences, Udaipur for their help during the study. Author declares no conflict of interest.

\section{References}

1. O'kaine M. The reporting, classification and grading of quality failures in the medical laboratory. Clin Chem Acta. 2009;404:28-31.

2. O'Kane MJ, Lynch PLM, McGowan N. The development of a system for reporting, classification and grading of quality failures in the clinical biochemistry laboratory. Ann Clin Biochem. 2008;45:129-134.

3. Reason JT, Carthey J, de Leval MR. Diagnosing 'vulnerable system syndromes': an essential prerequisite to effective risk management. Qual Health Care. 2001;10Supp12:ii21-5.

4. International Organization of Standardization. Quality Management System - Requirements. ISO 9001;2015, Geneva; 2015.

5. Witte DL, VanNess SA, Angstadt DS, Pennell BJ. Errors, mistakes, blunders, outliers or unacceptable results: how many? Clin Chem. 1997;43:1352-6.

6. Lapworth R, Teal TK. Laboratory blunders revisited. Ann Clin Biochem. 1994;31:78-84.

7. Hofgartner WT, Tait JF. Frequency of problems during clinical molecular genetic testing. Am J Pathol. 1999; 112:14-21.

8. Plebani M, Carraro P. Mistakes in a stat laboratory: types and frequency. Clin Chem. 1997;43:1348-51.

9. Reason J. Safety in the operating theatre - part 2: human error and organisational failure. Qual Saf Health Care. 2005;14:56-61.

10. Viera AJ, Garrett JM. Understanding interobserver agreement: The kappa statistic. Fam Med. 2005;37(5):3603.

11. Abdollahi A, Saffar H, Saffar H. Types and Frequency of Errors during Different Phases of Testing At a Clinical Medical Laboratory of a Teaching Hospital in Tehran, Iran. North American Journal of Medical Sciences. 2014;6(5):224-228. doi:10.4103/1947-2714.132941.

12. Howanitz PJ, Walker K, Bachner P. Quantification of errors in laboratory reports. Arch Pathol Lab Med. 1992;116:694700 .

13. Zemlin AE.Errors in the Extra-Analytical Phases of Clinical Chemistry Laboratory Testing. Indian J Clin Biochem. 2018;33(2):154-162.

14. AS Sakyi, EF Laing, RK Ephraim, OF Asibey, OK Sadique. Evaluation of analytical errors in a clinical chemistry Laboratory: A 3 year Experience. Ann of Medical Health Sci Res. 2015;5(1):8-12.

How to cite this article: Sadariya B. R, Sudhakar B. A systematic approach to report, categorize and grade quality failures in clinical biochemistry laboratory - 'single center analysis'. Int J Clin Biochem Res. 2018;5(4):617621. 